\title{
Desarrollo de las Ciudades en América Latina: ¿Qué forma de desarrollo sostenible es posible?*
}

\author{
Corbella, Oscar Daniel \\ Universidad Federal de Río de Janeiro, Brasil \\ oscar.corbella@gmail.com \\ Pérez Lobato, Nidia Iliana \\ Universidad de Xalapa, México
}

\author{
D'Avila Siqueira Neto, Gustavo \\ Universidad Federal de Rio de Janeiro, Brasil \\ gdsnmail@gmail.com
}

Resumen - Este trabajo propone una discusión sobre la dialéctica en el reconocimiento de la sostenibilidad como un nuevo modelo de desarrollo frente a la lógica moderna dominante. En el entendimiento de que debemos construir un nuevo paradigma, delante de la inevitable presencia de los riesgos ambientales y sociales en las ciudades. Esta discusión se transporta a la sostenibilidad de las ciudades en América Latina, a fin de promover una reflexión sobre el uso de la tecnología en su desarrollo idiosincrásico.

Palabras clave - Desarrollo Sostenible; Postmodernidad; Dialéctica; América Latina; Ciudades Sostenibles.

\begin{abstract}
This work proposes a discussion on the dialectic in the recognition of sustainability as a new development model, as opposed to the dominant modern logic. Understanding that we must build a new paradigm, in the face of environmental and social risks inevitable present in the cities. This discussion is transported to the sustainability of cities in Latin America, in order to promote a reflection on the use of technology in its idiosyncratic development.
\end{abstract}

Keywords - Sustainable Development; Postmodernity; Dialectics; Latin America; Sustainable Cities.

\section{ISSN: 2448-8704}

* Este trabajo fue originalmente publicado en francés en: Innovations technologiques et diversité culturelle (III) : Nouvelles perspectives pour le développement durable ? L'exemple des nanotechnologies. Proceedings of $3^{a}$ Édition du Séminaire international Innovations technologiques et diversité culturelle. Tema: Nouvelles perspectives pour le développement durable ? - L'exemple des nanotechnologies. Quebéc - Canada: Les Presses de l'Université Laval, 2016. p.133-146 


\section{LA DIALÉCTICA DE LA}

\section{SOSTENIBILIDAD: UN CAMINO}

INCOMPLETO ENTRE EL

\section{PRESENTE Y EL FUTURO.}

Cuando observamos la dialéctica del desarrollo sostenible desde una perspectiva social, política, económica y científica, podemos ver con mayor claridad que su razonamiento sugiere una temporalidad y espacialidad bipolar: las generaciones presentes y futuras, las naciones del norte y del sur, lo global y lo local, etc. Es aquí donde se refleja la existencia de dos polos separados, que no aparentan tener un espacio o tiempo intermedio, en el camino que se va construyendo, entre ellos; en esos residuos dialógicos que se van formando delante de la incertidumbre de sus modelos.

Esta dialéctica es inherente al concepto de desarrollo sostenible desde su inicio. Ya podemos observar, en 1987 (15 años desde la creación del Programa de las $\mathrm{Na}$ ciones Unidas para el Medio Ambiente (PNUMA) en Estocolmo -1972), cuando la Comisión Mundial de las Naciones Unidas sobre el Medio Ambiente y el Desarrollo pre- sentó el Informe Brundtland, también llamado "Nuestro futuro común", cuyo concepto se estableció como una alternativa de desarrollo que satisficiera las necesidades actuales de las naciones, sin comprometer a las generaciones futuras (Brundtland, 1991). Creando, así, en sus cimientos, el primer (de dos) camino incompleto que reconoceremos en este trabajo: el camino entre las acciones indispensables en el presente para saciar nuestras necesidades de desarrollo y los resultados futuros sin garantías. En este momento histórico, la idea de la sostenibilidad está aún sujeta a una lógica de acciones e intervenciones prácticas y momentáneas (apoyados en gran medida por los "expertos" internacionales), que, paradójicamente, solo produciría resultados más o menos precisos en el futuro, contando con la estabilidad de una condición político-económica específica. Lo que se torna imposible de obtener, especialmente frente a un destino desestabilizado por la amenaza de los cambios climáticos acelerados (Roaf et al., 2009). En esta perspectiva, parece que (en el campo de las acciones) uno de los principales problemas para el desa- 
rrollo de políticas efectivas para la sostenibilidad sigue siendo la eminencia de los riesgos ambientales, delante de la cualidad remota (no inmediata) de resultados que podrían obtenerse. El desfase entre el pasado, el presente y el futuro (lo que ha sido, lo que es y lo que podría ser) en la definición de prácticas sostenibles es, sin duda, un obstáculo importante para el reconocimiento de la sostenibilidad como principio indiscutible. Cada práctica o política en este sentido, parece obligada a pasar por un proceso de legitimación o "deslegitimación", bajo la visión crítica de otras áreas más "reconocidas", tales como la biología y la ecología, por ejemplo. La sostenibilidad ha podido hablar así de la supervivencia de la especie humana en el planeta, en nombre de las "ciencias universales". Según Henri Ascelrad (2001), esta incertidumbre con respecto a los resultados de las acciones actuales abre el debate ideológico en diversas áreas, de tal manera que no puede haber una sola respuesta.

La existencia del principio de la incertidumbre en la dialógica de Edgar Morin (1999) crea una oportuni- dad para cuestionar y replantear las relaciones de polaridad de los sistemas complejos, indo más allá de los modos de producción de conocimiento que reducen el todo a sus elementos esenciales. La dialógica entiende que un sistema complejo está integrado por las relaciones entre sus partes, que apuntan las reconstrucciones de las expresiones, narrativas, memorias y experiencias -partícipes de la dinámica social-, centrándose en el momento del contacto (reconocimiento) entre las partes como fundamental para la existencia del sistema. En ese momento de contacto se genera la incertidumbre, la cual, según Boaventura Santos (2011), ocurre por la traducción del significado que el fenómeno posee para cada parte. En ese momento de contacto-traducción, un fenómeno explica el otro por su concordancia o antagonismo. Esa condición de incertidumbre es indispensable para la transformación. Sin ella, los modelos serian cerrados. Es justo decir, que solo es posible realizar cambios en los sistemas complejos, porque ellos no son perfectos, porque existe la incertidumbre, que impide de percibirlo como totalizado -finalizado. Siempre habrá la incer- 
tidumbre: un área que no puede ser enteramente definida o traducida. Ella hace que los sistemas sean sanos. La creencia en la comprensión total de un fenómeno o en la palabra incuestionable, recae peligrosamente sobre las ideas absolutas de los padrones fundamentalistas.

La idea de que el desarrollo sostenible no es un estado que se debe alcanzar, sino un proceso, resuelve en parte el problema inicial sobre el camino recorrido entre el presente y el futuro, y se vuelve crucial para su comprensión. Inevitablemente, frente a la lógica del pensamiento dominante (y por falta de alternativas a ese), esta idea se vincula con el modelo de producción linear, que, según Arturo Escobar (2005a) es el mismo en sus bases, ya sea para una lógica de producción liberal o marxista. La sostenibilidad se convierte así en un proceso a implementar, basado en las experiencias y el conocimiento previo de los actores que ya están en la escena, sin una comprensión verdadera de que estamos delante de un nuevo y único paradigma, que requiere un esfuerzo de desconstrucción de ideas, para una reconstrucción sin modelos a priori.
Ella nace así con aspiraciones de "salvar a la humanidad" de una crisis global (especialmente con matices financieras), pero con pocas herramientas y cimientos para alcanzarla (Brunel, 2004).

\section{DIALÉCTICA DE LA}

SOSTENIBILIDAD: UN CAMINO

INCOMPLETO ENTRE EL "NOSOTROS" Y EL "OTRO".

La construcción de un concepto de sostenibilidad, basado en las herramientas, actores, lógicas y modelos coyunturales conocidos a priori, crea el segundo camino incompleto que se propondrá en este trabajo: el camino intermedio ignorado entre el "ellos" y el "nosotros" - entre los países "vendedores" de soluciones y los países "compradores". Al poner esta discusión en el contexto del occidente y particularmente con respecto a la América Latina, entendida en este texto como el "nosotros", buscamos comprender cómo la imagen de la América Latina ha sido creada y continúa siendo creada, como un lugar reconocido a través del "otro".

Como parte de su crítica de la modernidad occidental, Boaventura 
Santos (2010) explica la imagen de América Latina a través de la construcción de la propia identidad occidental moderna. Donde esta identidad se moldea inicialmente en la relación de alternalidad con el Oriente, visto en las cruzadas entre el siglo XI y XIII; posteriormente en la relación de inferioridad de sus colonias, entre el siglo XIV y XIX y finalmente en la relación de externalidad con respecto a la naturaleza, vista desde el surgimiento del iluminismo en el siglo XVII hasta los días actuales. Es así como podemos observar respectivamente tres factores de motivación: la fe, la modernidad y la técnica. Los tres presentes, a menudo por la fuerza, en la construcción de la Imagen de América Latina. La invención de la América Latina como identidad es referida por Walter Mignolo (2007) como una imposición histórica de las estructuras geopolíticas vigentes. Según el autor, después de que las naciones colonizadoras europeas se vieron obligadas a reconocer la autonomía de los estados ubicados en el norte de América (en el siglo $X(X)$, fue necesario reordenar la geografía política de Améri$c a$, dividiéndola entre anglosajona y latina. Es en este punto que se acuña la noción de latinidad, refiriéndose a los latinoamericanos como "europeos de segunda clase", excluyendo de esta latinidad a las poblaciones indígenas de América y las poblaciones afrodescendientes. Perpetuando así un proceso de servidumbre de la llamaba "América Latina", que gradualmente se trasladaría de la Europa para la América anglosajona. Cabe señalar que la bipolaridad geopolítica de esta relación de servidumbre es relevante para la comprensión de los modelos actuales de sostenibilidad, pero mítica para localizar espacialmente estos modelos en un eje norte-sur, este-oeste, etc., ya que las relaciones dominantes pueden darse desde cualquiera de los "lados", e incluso dentro de ellos. Con respecto a la sostenibilidad, no existe una polaridad real, pero sí, intencional.

En los procesos neocoloniales actuales, ya no estamos subyugados en nombre de la fe de los "conquistadores", pero aún estamos sometidos en nombre de la modernidad y la tecnología. Debemos aceptar proyectos de desarrollo que imponen técnicas y tecnologías entendidas como superiores, en nom- 
bre de una racionalidad que reprime nuestra identidad. En estos procesos hay una similitud con el colonialismo, por la intolerancia con la diferencia, por la creencia de un salvador y de un rescatado.

\section{MÚltiples}

SOSTENIBILIDADES: LA

BÚSQUEDA DE LA AUTORIDAD

\section{Y LA LEGITIMIDAD.}

Veinte años después de la Conferencia de Estocolmo, en la llamada Agenda 21 1992-, se realizó un cambio significativo en el sentido de la sostenibilidad, tratando de hacerla inclusiva (Sachs, 1992): EI término "desarrollo sostenible" adquirió su relevancia cultural actual, motivado por los movimientos sociales, cada vez más importantes, y por el creciente apoyo mediático. En un esfuerzo global para hacer del desarrollo sostenible un elemento socialmente incluyente, sostenible desde la perspectiva ambiental y económica; numerosos investigadores han intentado delimitar áreas de acción para este desarrollo, así como criterios operacionales para su funcionamiento. Entre ellos vemos la obra de Ignacy Sachs, que, a lo largo de varios años, delineó ocho perspectivas de organización del tema (Sachs, 2000): primeramente, social, cultural, ecológico, territorial, económico y ambiental, y más tarde, política nacional y política internacional. Estas áreas nos permiten entender que el discurso sobre el desarrollo sostenible tiene la característica de pasar a través de diferentes áreas a la vez, con diversas escalas y matices. Esta complejidad resulta en una dificultad por definir acciones y límites para el desarrollo. La cuestión tecnológica, que está claramente incluida en estas áreas, ha ganado mayor importancia en los últimos años, con su sorprendente progreso (particularmente en las áreas de nanotecnología, biotecnología y tecnología de la información). Esto podría, según Gilles Berhault (2010), ser clasificado como una perspectiva independiente del tema.

Más allá de la existencia de varias áreas de organización que, combinadas, hacen posible la construcción de "múltiples sostenibilidades", vemos que en nuestra sociedad permanecen diferentes discursos sobre el desarrollo sostenible. Esto establece diferentes modelos ideológicos para observar el mismo fenómeno. En este sentido, 
Wolfgang Sachs (1996) sugirió tres perspectivas para la comprensión del discurso sobre el desarrollo sostenible que difieren en la forma como implícitamente se entiende la finitud de los recursos del planeta: "la perspectiva fortaleza", "la perspectiva del astronauta" y "la perspectiva del hogar". La primera perspectiva es una visión tecnocéntrica, en la cual se cree que la sostenibilidad se refiere a la eficiencia social y ambiental, para el mantenimiento del desarrollo entendido como crecimiento económico. Se refiere así a una permanencia del estatus quo del modelo de producción de los cornucopianos modernos. Según este pensamiento, los recursos del planeta se vuelven idealistamente inagotables, ya que el capital, la ciencia y la tecnología tienen un alcance inimaginable $y$ puede solucionar cualquier problema relacionado con la sostenibilidad. Esta idea se puede apreciar, por ejemplo, en el punto de partida de la Declaración de Estocolmo (Jiménez, 2009): "la legitimidad de la conquista de la naturaleza por el poder de la modernización". La segunda perspectiva es una visión ambientalista, que identifica al planeta y sus recursos como limitados.
Estos últimos son indispensables para la supervivencia de la especie humana en la tierra, su preservación se convierte en una prioridad sobre todos los demás intereses humanos. En esta perspectiva, las naciones deberían establecer su producción de acuerdo y en proporción a los recursos disponibles: obligando a las naciones más industrializadas a reducir su producción y permitiendo a los menos industrializados aumentar la suya. La tercera perspectiva presentada busca descentralizar el desarrollo, equilibrando el nivel de producción y consumo de las naciones a la capacidad de resiliencia de sus ecosistemas. Esta se refiere a un mundo neomaltusiano limitado, donde el desarrollo no se centra en la capacidad económica, ni en la preservación del medio ambiente, sino en los medios de subsistencia. Por lo tanto, la sostenibilidad no es sólo una cuestión de eficiencia, sino también una cuestión de suficiencia: límites cuantitativos y cualitativos para el crecimiento de un sistema cerrado, lo que nos conduce a las nociones fundamentales de escala y de resiliencia de los ecosistemas. 
En teoría, cualquier discurso sobre la sostenibilidad puede tener su legitimidad cuestionada, si sus prácticas parecen no seguir una lógica racional, si excluyen a las partes menos favorecidas en la mayoría de sus procedimientos, o si no parece servir a un interés común. En este contexto, la legitimidad se enfrenta a algunos problemas críticos tales como, por ejemplo, definir y delimitar las partes involucradas; definir qué es un interés común o quién tiene el poder de reconocerlo. En el campo de la política y de la economía, por ejemplo, es normal que un interés común entre en conflicto con intereses particulares. Pero en otras áreas puede ser más fácil imaginar, por ejemplo, que el derecho a la vida es un interés común, no solo empíricamente, sino moralmente superior a cualquier otro interés (individual o colectivo). Cualquier práctica o política en este sentido, parece tener que pasar por una legitimación concedida sobre la base de otros principios diferentes de la sostenibilidad. Ésta hablaría sobre la supervivencia de la especie humana en el planeta en nombre de otras "ciencias universales". Es a partir de esta suposición que obser- vamos intentos de legitimar la sostenibilidad sobre otras ciencias y disciplinas. En una sociedad, cuando se trata de supervivencia, el derecho colectivo prevalece sobre el derecho individual, de acuerdo con estándares establecidos sobre la base del conocimiento existente (Bauman, 2003). Las reglas sociales de supervivencia son sacrificios legítimos que afectan a todos dentro de un principio democrático, mientras que las reglas del estatus quo económico y político son privaciones que afectan a algunos. En la misma línea, David Harvey (1996) y Ulrich Beck (2001) advierten sobre la posibilidad de que la sociedad adopte ciertas posturas totalitarias legítimas (o legitimadas) que reducen la libertad individual frente a los crecientes riesgos humanitarios. Según Harvey (1996), si los modelos globales (de justicia política, economía, etc.) -incluso si son llamados universales- se imponen sobre los modelos locales, se trata de una actitud autoritaria, impuesta a los menos favorecidos; del mismo modo, si los modelos locales suplantan a los modelos globales, sería una actitud autoritaria y xenófoba. Por lo tanto, se debe encontrar un modelo que permita alentar 
a la comunidad local y comprender su diversidad ( $\sin$ sobreestimarla), generando de esta manera el equilibrio y la equidad entre las partes. Un modelo que elabore un nuevo lenguaje, de una nueva lógica trinaría transdisciplinaria (Basarab $\mathrm{Ni}$ colescu, 2000), tomando en cuenta la información entre varias áreas de conocimiento, donde no solo hay falsos y verdaderos, y de nuevos conceptos que permitan un dialogo genuino entre las áreas.

\section{LA SOSTENIBILIDAD DE LAS CIUDADES}

De acuerdo con el informe de la ONU-Hábitat (2013), más de la mitad de la población mundial vive en zonas urbanas y este número sigue creciendo: en 2030, casi el $60 \%$ de la población vivera en las ciudades. A esto se suma el hecho de que estas ciudades, más allá de serán pensadas como nuestro nuevo Hábitat natural, están siendo construidas como los grandes íconos del "estilo de vida moderno". En ellas se concentra una gran parte de las acciones antropogénicas que son reconocidas como agentes significativos del cambio climático global (Roaf, 2010). Esta exaltación de la vida moderna en las ciudades sola- mente puede mantenerse mediante el uso indiscriminado de: a) tecnologías que sustituyen o reducen las labores que dependian antes de que un estilo de vida menos consumista; b) tecnologías que controlan el clima interno al permitir la capacidad de vivir en condiciones ambientales extremas para el ser humano, y c) tecnologías que automatizan funciones cada vez más complejas en los grandes centros urbanos. Estas tecnologías dependen de recursos energéticos caros y limitados y, siguiendo una lógica consumista, son rápidamente desechables, lo que aumenta la producción de residuos urbanos. En este contexto, las ciudades se convierten en el escenario más obvio de la lucha por la supervivencia de la humanidad, destacando las interconexiones entre las estructuras urbanas, sus construcciones y el ecosistema global.

Para llevar la discusión del desarrollo sostenible al tema urbano, Ascelrad (2001) presenta varias matrices discursivas existentes sobre la sostenibilidad urbana. Según el autor, la ciudad puede, en principio, ser representada de tres maneras: 1) la ciudad como objeto que 
representa un material técnico y complejo que obedece a principios físicos 2) la ciudad considerada como un espacio donde hay operaciones cuyo objetivo final es la "calidad de vida", y 3) la ciudad como un lugar para reconstruir la legitimidad de las políticas urbanas. Escenario del poder de las instituciones públicas y privadas $y$, en particular, de la responsabilidad del Estado. La cuestión tecnológica se destaca en la primera representación, por la subordinación a la que está sujeta la ciudad. En esta representación, el autor plantea dos modelos: a) un modelo de racionalidad eco-energética. Es decir, la representación técnico-material de la ciudad como matriz compuesta por un vector de consumo (de espacio, energía y materiales) y otro vector de producción de residuos, por un mismo rango de servicios. b) Un modelo de equilibrio metabólico (originalmente propuesto por Herbert Girardet). Es decir, la representación ecosistémica de las ciudades, compuesta por movimientos de circulación interdependientes, intercambios y transformación de recursos. Un adecuado ajuste de las reservas y flujos de materia y energía.
En términos de diseño y planificación urbana, su sostenibilidad puede ser mejorada por su forma, tamaño, configuración y distribución espacial. Por lo tanto, las relaciones menos dispares entre la producción y el consumo local, entre la entrada de recursos y la salida de residuos, así como la reducción de los flujos (energéticos, materiales y económicos) en la ciudad, pueden promover un metabolismo más equilibrado. Estos problemas se traducen en una forma urbana más equitativa. Las nuevas tecnologías pueden tener un papel relevante en este metabolismo, por ejemplo, potenciando el uso de fuentes de energía renovables, reduciendo la necesidad de recursos materiales y humanos, o reduciendo el desperdicio y las pérdidas en los procesos que involucran a la ciudad.

Si, por un lado, se puede pensar que el uso eficiente de los recursos medioambientales y la calidad de vida urbana son atributos de las ciudades poco densas, permitiendo la producción local de recursos (entradas) en el suelo disponible; por otro lado, también se puede pensar que son atributos de las ciudades densas, pues concentran el consu- 
mo y el desperdicio. En este caso, hay una intensificación, que aumenta la eficiencia energética y material para reducir, reutilizar o reciclar. En la práctica, la condición ideal del metabolismo urbano, con entradas mínimas, flujo interno reducido y bajas salidas no puede cumplirse en ningún modelo posible de ciudad. La eficiencia consiste en encontrar un estado donde la "falta" en el metabolismo ideal se ve más o menos compensada por la "abundancia". No solo en términos cualitativos, sino también en términos cuantitativos.

Cabe señalar que la cuestión de la sostenibilidad de la ciudad también debe abordarse mediante la capacidad de gestionarla democráticamente. Refiriéndonos a problemas tales como la densidad de la ciudad y la disponibilidad de recursos que guiarán la estrategia que se utilizará. $\mathrm{Si}$, por ejemplo, por un lado, la densificación permite un mejor uso del suelo, por otro lado, conduce a una sobrecarga en términos de infraestructura y de consumo de recursos. Demandando sistemas tecnológicos más grandes y mejores para mantener sus estructuras en funcionamiento. Es así como, lo que podría ser adecuado para países densamente poblados como los de Europa no lo sería necesariamente para países con una extensa área territorial, como Brasil, Estados Unidos o Canadá. Las soluciones menos densas y más pequeñas que se conectan a una red de ciudades podrían, por ejemplo, ser una alternativa más sostenible para las ciudades latinoamericanas. Los llamados modelos de sostenibilidad universal se han construido en una extraterritorialidad que no se puede aplicar de la misma manera a las diferentes condiciones territoriales y demográficas. La pregunta principal es la definición de la solución más efectiva para cada situación, teniendo en cuenta, además de la condición territorial, la accesibilidad técnicoeconómica de las poblaciones, las diferencias climáticas y la diversidad cultural, que recaerán sobre el "equilibrio ecológico". Esta cuestión territorial es amplificada por Escobar (2005b) hasta una cuestión ecosistémica, donde la sostenibilidad consiste en una articulación de los proyectos de vida de las comunidades con el proyecto político de los movimientos sociales, en el espacio que él llama territorio-región. 
Por lo tanto, cada ciudad debe tener una solución adaptada a su territorio, sus recursos, sus medios de producción, su clima y su cultura.

Considerando a la ciudad como un concepto transitorio, porque se encuentra constantemente transformación (Ascher, 1995), entendemos que la ciudad sostenible no puede existir como un fin en sí misma, sino como un proceso continuo. Entonces, la mutación natural de las ciudades debe conducir a una valoración de las acciones en detrimento del resultado. Cualquier producto que se dice ser sostenible y que no valore el proceso, sólo da como resultado un pastiche de sostenibilidad. Como se puede ver en muchos proyectos nuevos (urbanos y arquitectónicos), que, debido a que hablan por el bien de la tecnología, pretenden ser sostenibles. Esta creencia en la capacidad de la ciencia moderna para superar los riesgos derivados de las actividades humanas se queda en el discurso ProDesarrollo y se pueden observar en muchos "ecodiseños" futuristas del imaginario arquitectónico y urbanístico.
En lo que respecta a las edificaciones, en la actualidad el concepto comercial de un edificio moderno es el de un edificio con tecnología aplicada. Nuevas tecnologías (industrializadas) que se usan en detrimento de las tecnologías tradicionales, que podrian existir como alternativas. Un error común y grave es pensar que un edificio moderno sería sinónimo de un edificio sostenible, que cuanto más avanzada sea su tecnología, más sostenible será. En este campo, a menudo vemos nuevas tecnologías, que pretenden reemplazar una tecnología más antigua, considerada obsoleta. Una vez más, se debe tener cuidado sobre quién tiene la autoridad para rechazar una tecnología en beneficio de otra. En ocasiones, algunas de estas tecnologías pueden eventualmente abandonarse por razones políticas o económicas muy alejadas de las razones de la eficiencia o la suficiencia. También debemos poner atención a la dependencia técnica y económica que estas nuevas tecnologías pueden generar. Es importante recordar que la discusión sobre la sostenibilidad de los edificios ahora está distorsionada. Ella valora el respeto por una serie de 
elementos "ambientalmente correctos" de una lista de nuevas técnicas y tecnologías impuestas por el mercado. Podemos observar, por ejemplo, un gran número de códigos y etiquetas de calidad, creados recientemente para el campo de la construcción, que normalizan indiscutiblemente el sector, pero no garantizan la sostenibilidad de las construcciones. A nivel mundial, ha habido una demanda creciente de sistemas de tecnologías más "amigables" con el medio ambiente, lo que ha resultado en la integración de nuevas tecnologías para los edificios. Particularmente, la nanotecnología trajo una transformación significativa en la producción de nuevos materiales de construcción, mediante el aumento o la modificación de sus características físicas. Según Roaf, (2010) el mundo tiende a avanzar hacia la eficiencia de estos sistemas tecnológicos en las edificaciones, pero los principales obstáculos siguen siendo las fuerzas del mercado, los organismos reguladores, la legislación y la gestión política del problema. Debido a que la tecnología, en particular la nanotecnología, no es inofensiva: ella plantea cuestiones de carácter económico, medioambiental e inclu- so moral. La búsqueda exaltada de eficiencia ofusca la cuestión inevitable de la suficiencia. Para un futuro sostenible, además de centrarnos en mejorar los sistemas tecnológicos, debemos ir más allá al cambiar el comportamiento del mercado, del estado y de la sociedad.

\section{REPRESENTACIONES Y DECONSTRUCCIONES}

La modernidad occidental parece jugar un papel importante en la construcción de la imagen de América Latina. Así nos encontramos con varios autores (Escobar, 2005b; Mignolo 2007; Santos, 2010) que reconocen al colonialismo como un componente esencial de esta modernidad. Según estos autores, existe una colonialidad que es parte de la modernidad y que no puede existir sin ella. Al ir en contra de la idea de que la modernidad es un proyecto inconcluso que debe completarse, estos autores proponen una reinvención de la modernidad desde una perspectiva poscolonial. Santos (2010) argumenta que, al excluir el colonialismo de la autorrepresentación de la modernidad, se creó una imagen incompleta de esta última y sus fundamentos. 
Abriendo una vía para procesos no perceptibles (no reconocibles por la ligereza de su identidad) que conducen al colapso de la modernidad misma. El autor enfatiza la importancia de la autorrepresentación para comprender las relaciones de poder actuales a escala global. Posiciona la actual relación "norte-sur" en una coyuntura histórica donde las dos partes se "descubren"': una como salvadora y otra como rescatada. Lo que a primera vista puede parecer solo un hecho histórico, tiene, hasta hoy, considerable relevancia en la forma en que nos vemos a nosotros mismos (en el "sur") y nos representamos, reflejados en el "norte". Entonces, si entendemos que nuestras referencias, a priori para la construcción de un concepto de sostenibilidad, se basan en los mismos valores de la modernidad, independientemente de la relación norte-sur, podremos ver que la discusión sobre la sostenibilidad va más allá de una dicotomía entre el más y el menos favorecido. Va al cuestionamiento de nuestro modelo de civilización.

Para algunos autores, como Harvey (1996), el debate sobre la sostenibilidad es un debate acerca de un orden preestablecido: el modelo de producción capitalista. Tal lectura nos permite entender el fenómeno, considerando la permanencia del estatus quo del modelo de producción, sin discutir una posible quiebra de los paradigmas actuales de producción. El desarrollo de nuevas tecnologías y su capacidad para alterar la suficiencia y la eficiencia de los sistemas nos sorprende cada día y puede llevarnos a la tentación de creer que todo es posible. Sin embargo, definir escalas y límites es fundamental para la sostenibilidad de un mundo limitado. No obstante, tal idea entra en conflicto con nuestro paradigma actual. Porque "hablar de límites implosiona la promesa liberal de la abundancia universal por el crecimiento de la demanda y de un mercado en constante expansión" (traducción libre - Acselrad 2009, p.49). No debemos dejarnos seducir tampoco por la promesa de que las tecnologías resolverán todas las externalidades del modo de producción actual. Hay una transición que debe hacerse: las nuevas tecnologías no deben ser utilizadas para apoyar los modelos sobrepasados, más bien para hacer posible la existencia de nuevos modelos. Si 
creemos que la sociedad está pasando por una transformación fundamental, que algunos autores, como Beck (2001) y Touraine (2011) llaman "crisis" de los modelos actuales, ¿no sería posible pensar en un desarrollo según un modelo diferente al actual?

Esta creciente percepción de crisis, la insatisfacción con el desarrollo de los países "menos favorecidos" y el modelo de sostenibilidad de los países "más favorecidos", así como la aparente incapacidad de la ciencia y la tecnología para resolver todos los problemas sociales, económicos y ambientales de la modernidad, sugieren la necesidad de una nueva forma de desarrollo, citada por algunos críticos como el posdesarrollo (Escobar, 2005). En este sentido, diversos críticos de la modernidad (Bauman 1998; Giddens, 1991; Santos, 2010) reconocen que existen presiones sociales y culturales actuantes, que obligan al actual modelo civilizatorio -cuyo el desplome es cada vez más evidente- a hacer una transición hacia paradigmas emergentes, reconociendo la necesidad de reconstrucción de los modelos actuales. Sugiriendo que la perspectiva poscolonial transciende a la modernidad occidental, Santos (2010) apunta que es necesario recrear la democracia, la política e incluso el Estado, con base en lo que él llama una "epistemología del Sur" (Santos, 2011). $Y$ esto, entendiendo al Sur no como un lugar geográfico, sino como una realidad sociopolítica de quienes sufren con el neocolonialismo. Un nuevo modelo de desarrollo, en el que la naturaleza y la sociedad sean percibidas como una sola cosa. En el que, de acuerdo con los conceptos posmodernos, la naturaleza no es externa al hombre: es parte de, no pudiendo ser separados o percibidos de una manera diferente.

\section{LA REINVENCIÓN DEL}

\section{PARADIGMA.}

Las ciudades, sus estructuras y el estilo de vida de sus habitantes están en el centro de la cuestión de la sostenibilidad. Hoy en día tenemos que reducir la generación de contaminantes y residuos, aumentar el bienestar en las ciudades mediante la reducción de los costos de energía, extender la vida útil de los edificios, respetando el metabolismo natural de las ciudades y la ca- 
pacidad de recuperación de sus ecosistemas. Tenemos que crear nuevas ciudades y replantear las antiguas, en una perspectiva más amplia que la del mercado, teniendo en cuenta también su límite de gestión democrática. Es necesario crear sistemas técnicos y tecnológicos para desarrollar y mantener estas condiciones, en la misma forma que los recursos del planeta. Las nuevas tecnologías aplicadas a las ciudades deben cumplir un papel central en la eficacia de estos sistemas, sin dejar de lado la suficiencia. Siempre reforzando el hecho de que cualquier sistema tiene un límite y que operar más allá de este límite, incluso si es en nombre de la eficiencia, conduce a un fallo del sistema.

A nivel mundial, después de que han pasado más de 40 años desde que se dieron los primeros debates sobre cuestiones ambientales globales, la idea de desarrollo sostenible sigue en proceso de maduración, en particular con respecto a su inclusión. Sus caminos aún presentan algunos vacíos por llenar, a pesar de los muchos avances obtenidos. La responsabilidad en la solución (que debe ser de to- dos), no ha sido aún reconocida; tal vez porque todavía buscase el "gran responsable" del problema, cuando en realidad el no existe como tal, ya que es una parte inseparable de la lógica moderna. A pesar del planteamiento, aparentemente inalcanzable, que el concepto de desarrollo sostenible puede presentar, es la expresión más legítima de la responsabilidad del hombre en relación con la existencia. Aquí, las diferentes representaciones y valores, ya sea una perspectiva global - local, buscan el reconocimiento y la aceptación de sus cánones. En sus diversas orientaciones ideológicas (por no decir existenciales), el desarrollo sostenible siempre se coloca como la esperanza de la redención de la humanidad. Si bien que los fundamentos de la racionalidad económica, la idolatría técnico-científica y la universalización de bien social, no hacen más que aumentar las desigualdades e insuficiencias del planeta, en nombre de esta "causa" que busca legitimar (con diferentes nombres) la continuidad del modelo la producción actual. En este contexto, la sostenibilidad sigue siendo un proceso construido a partir de modelos insuficientes de desarrollo. Sus fallas 
no pueden ser solventadas o resueltas en el seno de estos modelos. La sostenibilidad requiere un esfuerzo de deconstrucción de sus fundamentos para lograr una reinvención inocua. Un momento de inflexión sobre sus paradigmas, en los que encontramos los discursos basados en la utopía o la catástrofe.

En la búsqueda de un modelo de sostenibilidad para las ciudades de América Latina, hay que entender primero su identidad con el fin de promover la comunidad local y su diversidad. Las tecnologías -sus valores y usos- deben concebirse en esta identidad y no a la inversa. América Latina, tal y como es reconocida, como lugar, a través del "otro", no puede realmente hacer parte de esta ecuación.

Es necesario romper estas condiciones de bipolaridad creados por la modernidad y generar un verdadero equilibrio y una equidad entre las partes. Mientras América Latina no se redescubre, cabe cuestionar si la condición de latinidad es un destino fatídico, del que no se puede escapar. ¿Es que siempre se requiere dejar nuestras venas abiertas (en referencia a la obra de Eduardo Galeano, 2010) a la compasión de los más hegemónicos?

\section{REFERENCIAS}

Acselrald, H. (2009). Sentidos da Sustentabilidade Urbana. En H. Acselrald (org), A duração das cidades:

sustentabilidade e risco nas políticas urbanas (pp. 43-70). Rio de Janeiro: Lamparina Editora.

Ascher, F. (1995). Metapolis: ou l'avenir des villes. Paris: Odile Jacob.

Bauman, Z. (1998) O mal estar da pós-modernidade. Rio de Janeiro: Jorge Zahar.

Bauman, Z. (2003) Comunidade: a busca por segurança no mundo atual. Rio de Janeiro: Jorge Zahar. Beck, U. (2001) La Société du Risque- Sur la voie d'une autre modernité. Paris: Aubier.

Berhault, G.(2010) Développement durable 2.0: l'internet peut-il sauver la planète?. Paris: Éditions de l'Aube. Brundtland, G. H.; et al. (1991) Nosso Futuro Comum - Comissão Mundial sobre o Meio Ambiente e Desenvolvimento. Rio de Janeiro: FGV.

Brunel, S. (2004) Le développement durable. Paris: Presse Universitaire de France. 
Escobar, A. (2005a) El "postdesarrollo" como concepto y práctica social. En D. Mato (coord.), Políticas de economía, ambiente y sociedad en tiempos de globalización (pp. 17-31). Caracas: Facultad de Ciencias Económicas y Sociales, Universidad Central de Venezuela.

Escobar, A. (2005b) Más allá del Tercer Mundo Globalización y Diferencia. Bogotá: ICANH.

Galeano, E. (1996) As veias abertas da América Latina. Rio de Janeiro: Editora Paz e Terra.

Giddens, A. (1991) As consequências da modernidade. São Paulo: Editora da UNESP.

Harvey, D. (1996) Justice, Nature and the Geography of Difference. Oxford: Blackwell.

Jiménez, M. E. S. (2009) Sustentabilidad a dos tiempos. Polis, Revista de la Universidad Bolivariana, 24(8), 357-382.

Mignolo, W. D. (2007) La idea de América Latina: La herida colonial y la opción decolonial. Barcelona: Editorial Gedisa.

Morin E. (1999). Los siete saberes necesarios para la educación del futuro, Paris: UNESCO.

Nicolescu, B. (2000) Transdisciplinarity and Complexity - Levels of Reality as Source of Indeterminacy, Niveaux de Réalité (I), Rencontres Transdisciplinaires, Bulletin $n^{\circ} 15$,
UN-HABITAT (2013) The State of the World's Cities 2012/2013: The Prosperity of Cities. ONU.

Roaf, S. et al. (2009) A adaptação de edificações e cidades às mudanças climáticas. Porto Alegre: Bookman.

Sachs, I. (1992) Equitable Development on a Healthy Planet. Paris: EHESS, CIRED.

Sachs, I. (2000) Caminhos para o desenvolvimento sustentável. Rio de janeiro: Garamond.

Sachs, W. (1996) La anatomía política del "desarrollo sostenible". En ECOFONDO-CEREC, La gallina de los huevos de oro: Debate sobre el concepto de desarrollo sostenible(pp.15-43) Bogotá: Ecofondo-Cerec.

Santos, B. de S. (2010) A gramática do tempo: para uma nova cultura política. São Paulo: Cortez.

Santos, B. de S. (2011) Épistémologies du Sud. Études rurales, 187(1), 21-49.

Touraine, A. (2011) Apôs a crise: A decomposição da vida social e o surgimento de atores não sociais.

Petrópolis: Vozes. 